\title{
Spectral analyses of three carbon-enhanced metal-poor stars
}

\author{
N. T. Behara ${ }^{a b *}$, P. Bonifacio ${ }^{a b c}$, H.-G. Ludwig ${ }^{a b}$, L. Sbordone ${ }^{a b}$, \\ J. I. González Hernández ${ }^{a b}$ and E. Caffau ${ }^{b}$ \\ ${ }^{a}$ CIFIST Marie Curie Excellence Team \\ ${ }^{b}$ GEPI, Observatoire de Paris, CNRS, Université Paris Diderot, France \\ ${ }^{c}$ Istituto Nazionale di Astrofisica, Osservatorio Astronomico di Trieste, Italy \\ E-mail: natalie.behara@obspm.fr
}

Approximately $20 \%$ of very metal-poor stars $([\mathrm{Fe} / \mathrm{H}]<-2.0)$ are strongly enhanced in carbon $([\mathrm{C} / \mathrm{Fe}]>+1.0)$. Such stars are referred to as carbon-enhanced metal-poor (CEMP) stars. A variety of abundance patterns are found among CEMP stars. Strong overabundances of nitrogen are common, and overabundances of neutron capture elements are often, however not always, present. The variety of abundance patterns among CEMP stars strongly suggests that this population of stars comprises several astrophysical origins.

We are conducting a high-resolution follow-up of candidate EMP stars extracted from the Sloan Digital Sky Survey (SDSS; York et al. 2000) using UVES at the VLT. Three of the programme stars, SDSS J0912+0216, SDSS J1036+1212 and SDSS J1349-0229, where deliberately targetted as CEMP stars since a strong $G$ band was evident from the SDSS spectra and the weakness of the $\mathrm{Ca}$ II $\mathrm{K}$ line testified their very low metallicity. The UVES high resolution follow-up confirmed the original findings $([\mathrm{Fe} / \mathrm{H}]<-2.50)$ and allowed a more detailed investigation of their chemical composition.

We determined the carbon abundance from molecular lines which form in the outer layers of the stellar atmosphere. It is known that convection in metal-poor stars induces very low temperatures which are not predicted by classical 1D stellar atmospheres. To obtain the correct temperature structure, one needs full 3D hydrodynamical models. 3D carbon abundances were determined for all three stars, using $\mathrm{CO}^{5} \mathrm{BOLD} 3 \mathrm{D}$ hydrodynamical model atmospheres. 3D effects on the carbon abundance are found to be quite significant for these stars, with 3D corrections of up to -0.7 dex.

Two of the stars, SDSS J0912+0216 and SDSS J1349-0229 exhibit an overabundance of neutron capture elements which classifies them as CEMP-s. Star SDSS J1036+1212, instead belongs to the elusive class of CEMP-no/s stars, with enhanced $\mathrm{Ba}$, but deficient $\mathrm{Sr}$, of which it is the third member discovered to date.

10th Symposium on Nuclei in the Cosmos

July 27 - August 1, 2008

Mackinac Island, Michigan, USA

\footnotetext{
* Speaker.
} 


\section{Introduction}

The EMP candidates have been selected using a version of our automatic analysis code (Bonifacio \& Caffau 2003) tailored to SDSS spectra of turn-off stars which provides an estimate of $[\mathrm{M} / \mathrm{H}]$. Follow up high resolution spectra were acquired with UVES at the VLT, with a 1"! 4 slit and $2 \times 2$ on-chip binning, providing a resolution of $\sim 30000$. Figure 1 displays the $\mathrm{CH} G$ band of all three stars, showing clearly the $\mathrm{C}$ enhancement.

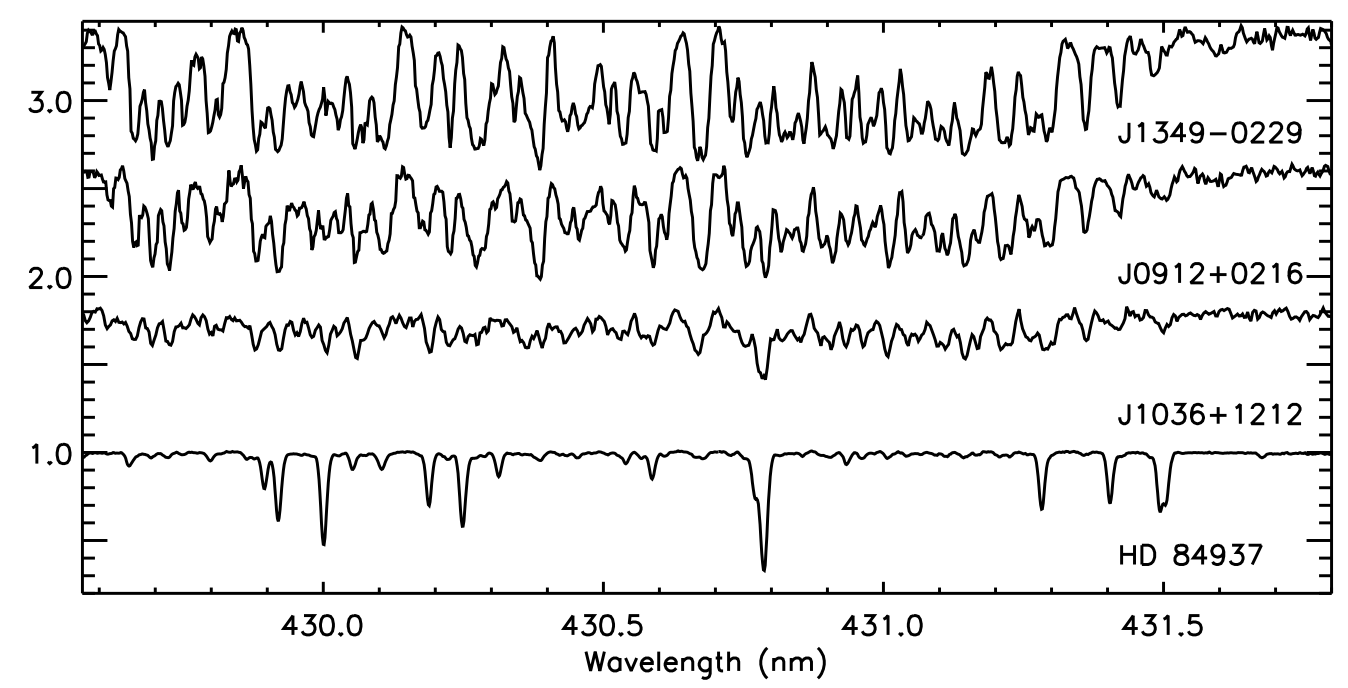

Figure 1: Observed spectra of the $\mathrm{CH} G$ band in the three CEMP stars, compared to the same spectral region of a dwarf metal-poor star, HD84937, showing no carbon enhancement. Spectra have been offset for clarity.

\section{Atmospheric parameters and analysis}

As a first estimate of the effective temperatures of our stars, we used the wings of $\mathrm{H} \alpha$. Temperatures were then redetermined using the Fe I excitation equilibrium, and were found to agree with the $\mathrm{H} \alpha$ temperatures to within $100 \mathrm{~K}$. The surface gravity was derived from the $\mathrm{Fe} \mathrm{I} / \mathrm{Fe}$ II ionisation equilibrium, and the microturbulence was determined by requiring Fe I lines to provide the same abundance regardless of line strength. Equivalent widths of $\mathrm{Fe}$ I and $\mathrm{Fe}$ II lines have been measured by means of the FITLINE code (François et al. 2003). ATLAS model atmospheres and SYNTHE (Kurucz 1993, 2005) synthetic spectra, in their Linux version (Sbordone et al. 2004, Sbordone 2005), have been employed in the analysis. Adopted stellar parameters are listed in Table 1 .

\begin{tabular}{lrlrrrrr} 
Star & $T_{\text {eff }}$ & $\log g$ & $\xi$ & {$[\mathrm{Fe} / \mathrm{H}]$} & {$[\mathrm{C} / \mathrm{Fe}]$} & {$[\mathrm{Ba} / \mathrm{Fe}]$} & {$[\mathrm{Sr} / \mathrm{Fe}]$} \\
\hline $\mathrm{J} 1349-0229$ & 6200 & 4.00 & 1.5 & -3.0 & $+2.17 \pm 0.04$ & $2.26 \pm 0.05$ & $+1.35 \pm 0.06$ \\
$\mathrm{~J} 0912+0216$ & 6500 & 4.50 & 1.5 & -2.5 & $+1.53 \pm 0.03$ & $1.58 \pm 0.04$ & $+0.53 \pm 0.07$ \\
$\mathrm{~J} 1036+1212$ & 6000 & 4.00 & 1.4 & -3.2 & $+0.98 \pm 0.13$ & $1.26 \pm 0.03$ & $-0.51 \pm 0.04$
\end{tabular}

Table 1: Adopted stellar parameters and derived abundances. Carbon abundances were corrected for 3D effects. 


\section{Carbon}

The $\mathrm{C}$ abundances derived from the 1D analysis were corrected for 3D effects (see Caffau et al. 2008, and references therein, for our definition of 3D corrections) using 3D model atmospheres computed with $\mathrm{CO}^{5} \mathrm{BOLD}$ (Freytag et al. 2002, Wedemeyer et al. 2004). The 3D spectral synthesis calculations were performed with the code Linfor3D. The isolated $\mathrm{CH}$ features at $416.4 \mathrm{~nm}, 416.9$ $\mathrm{nm}, 418.0 \mathrm{~nm}$, and $418.8 \mathrm{~nm}$ were used to determine $\mathrm{C}$ abundances. These lines form in the outer layers of the stellar atmosphere. 3D and 1D profiles of the $\mathrm{CH}$ line $416.4 \mathrm{~nm}$ are shown in the left panel of Figure 2. The contribution functions for this line, along with the model temperature distributions are shown in the right panel. The outer layers of the 3D atmosphere are much cooler compared to the 1D model, resulting in a much stronger line. Throughout the analysis, the oxygen abundance was fixed at $[\mathrm{O} / \mathrm{Fe}]=0.4$.
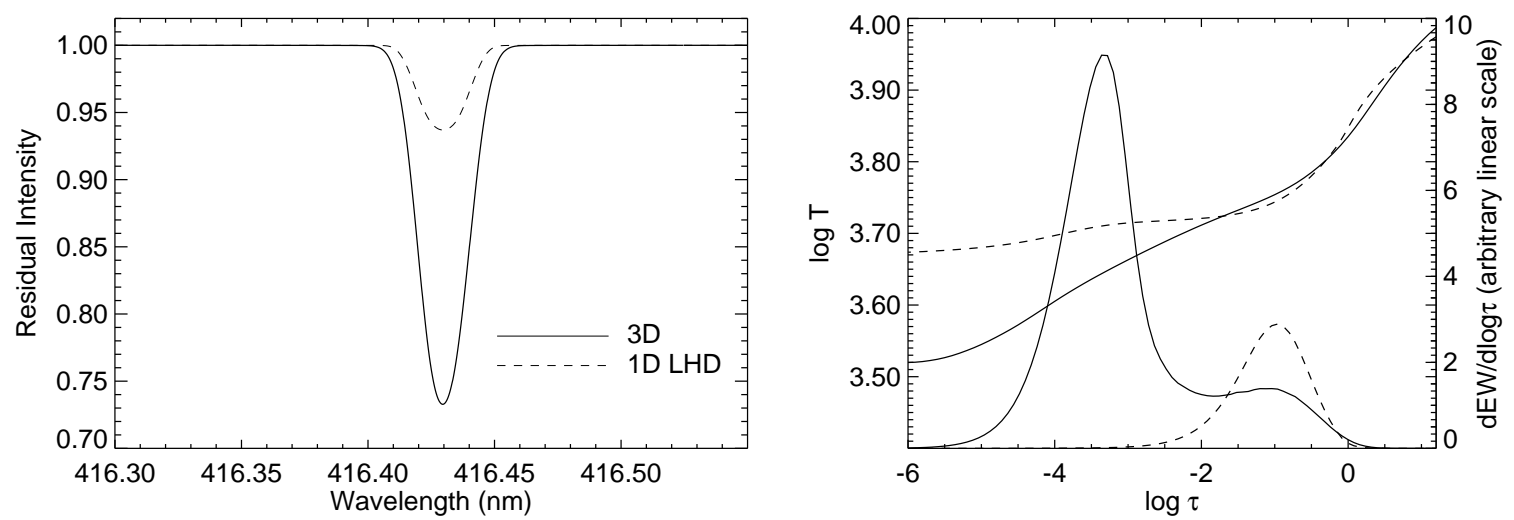

Figure 2: Left panel: 3D and 1D profiles of the $\mathrm{CH}$ line $416.4 \mathrm{~nm}$ computed using models with $\mathrm{T}_{\text {eff }}=6550 \mathrm{~K}, \log g=4.50$ and $[\mathrm{Fe} / \mathrm{H}]=-3.00$. Right panel: The contribution functions for the equivalent width of this line, along with the model temperature distributions. In both panels, the 3D model is plotted using a solid line and the 1D model using a dashed line.

There are two main effects that distinguish 3D from 1D models, the average temperature profile and the horizontal temperature fluctuations. We quantify the contribution of both effects by introducing the 3D correction as: 3D - 1D. The 3D corrections obtained for the three stars range from -0.5 to -0.7 dex, and have been already applied to the carbon abundances listed in Table 1 .

\section{Neutron-capture elements}

We derive strontium abundances for our stars using the Sr II $407.7 \mathrm{~nm}$ and $421.5 \mathrm{~nm}$ lines. The two stars with the highest carbon abundance exhibit overabundances of $\mathrm{Sr}$ from 0.5 to 1.35 dex, while the star with the lowest $\mathrm{C}$ abundance exhibits an underabundance of $\mathrm{Sr}$ by 0.5 dex. Barium is overabundant in all three stars. The Ba abundances were estimated from the Ba II $493.4 \mathrm{~nm}$ and $614.1 \mathrm{~nm}$ lines. For the analysis we adopted the hyperfine splitting by McWilliam (1998) and assumed a solar isotopic mix. The measured $\mathrm{Ba}$ and $\mathrm{Sr}$ abundances are listed in Table 11. The analysis was performed using 1D LTE models. 

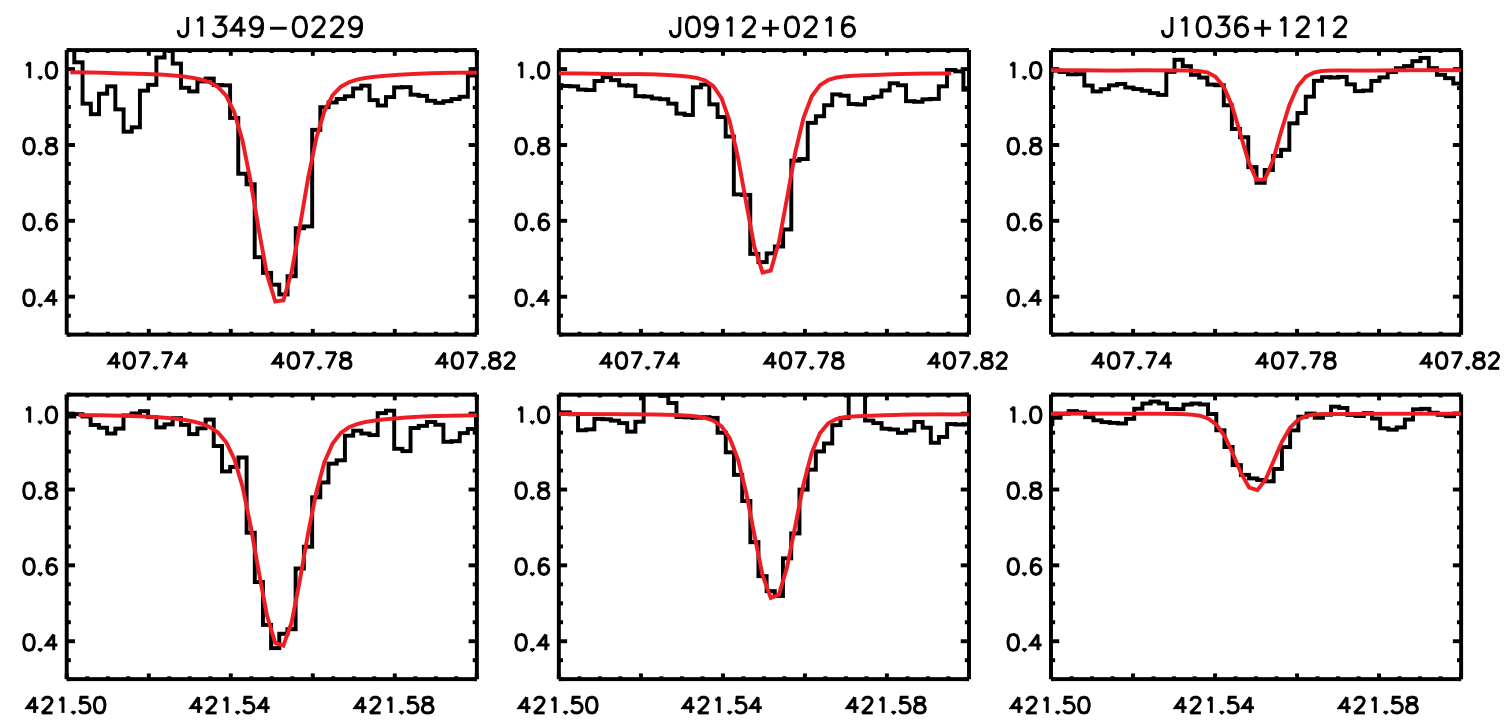

Figure 3: Sr II $407.7 \mathrm{~nm}$ and $421.5 \mathrm{~nm}$ lines used in the abundance analysis.

\section{Discussion and conclusions}

The 3D effects on the carbon abundance determined using $\mathrm{CH}$ lines were found to be significant, with $3 \mathrm{D}$ corrections of up to $-0.7 \mathrm{dex}$. This suggests that all the published $\mathrm{C}$ abundances in dwarf CEMP stars, based on molecular lines and 1D models should be reconsidered in the light of hydrodynamic simulations. Star SDSS J1349-0229 exhibits a strong $\mathrm{C}_{2}$ Swan band which classifies it as a carbon star $(\mathrm{C} / \mathrm{O}>1)$, while the other two stars are carbon-enhanced stars.

In Figure 7 we plot the $\mathrm{Sr}$ and $\mathrm{Ba}$ abundances of the three stars (red symbols) together with a sample of 24 CEMP stars from Table 4 of Sivarani et al. (2006). It is now widely accepted that the CEMP class includes objects which have rather diverse astrophysical origin. One sub-classification of such stars was proposed by Beers \& Christlieb (2005) based on the abundances of neutroncapture elements in CEMP stars. One therefore speaks of CEMP-no stars, when no enhancement of neutron capture elements is observed; CEMP-s when only the s-process elements are enhanced, and CEMP-r/s when both r-process and s-process elements are enhanced. The class CEMP-no/s was introduced by Sivarani et al. (2006) to contain the stars CS 29527-041 and CS 31080-095 which where overabundant in Ba but underabundant in Sr. Star SDSS J1036+1212 is the third star known to fall into this category. Interestingly all three known CEMP-no/s stars are warm dwarf/TO stars. The vast majority of CEMP dwarf stars is understood as the result of mass-transfer from a presently unseen companion which has undergone the AGB phase. The peculiar $\mathrm{Sr} / \mathrm{Ba}$ ratios of the CEMP-no/s stars should provide useful constraints on the properties of the companion star while on the AGB.

The other two stars, SDSS J0912+0216 and SDSS J1349-0229, exhibit both high abundances of $\mathrm{Sr}$ and $\mathrm{Ba}$, indicating that they are CEMP-s stars. Our single epoch observations offer no indications on the possible existence of binary companions, however radial velocity monitoring of all three would be highly desirable. 


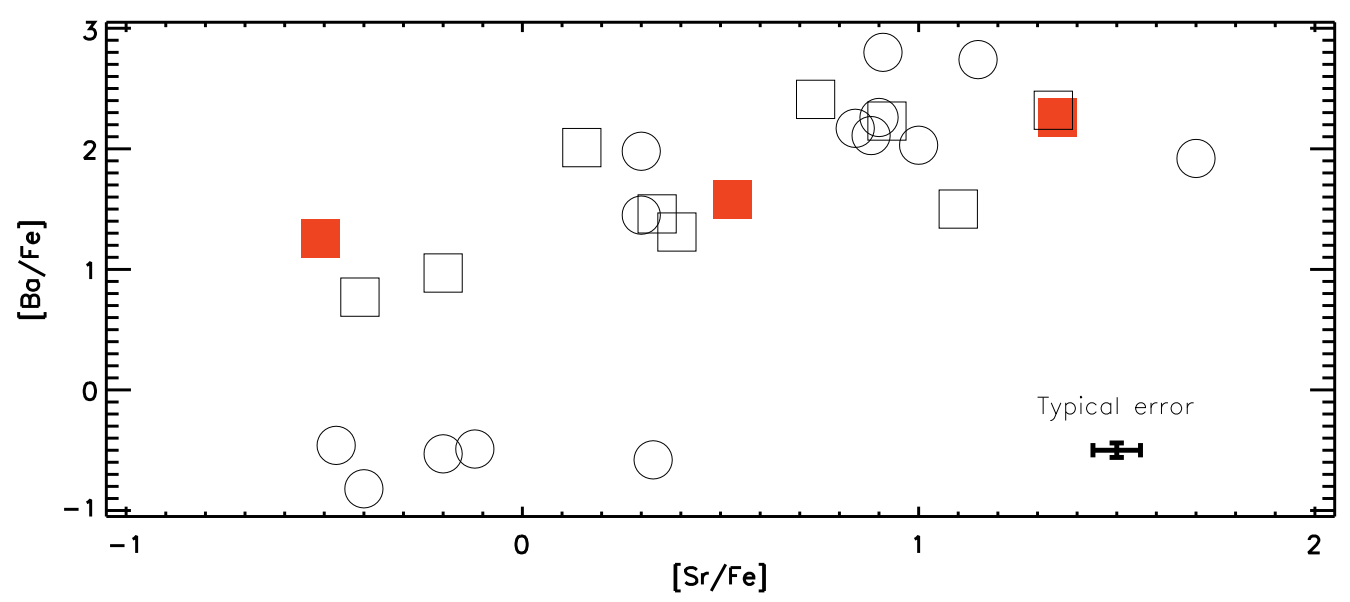

Figure 4: $[\mathrm{Ba} / \mathrm{Fe}]$ vs. [ $\mathrm{Sr} / \mathrm{Fe}]$ for our three CEMP stars (red filled symbols) plotted alongside values for 24 CEMP stars (open squares for dwarfs, circles for giants) listed in Table 4 of Sivarani et al. (2006).

\section{References}

[1] T. C. Beers,\& N. Christlieb, The Discovery and Analysis of Very Metal-Poor Stars in the Galaxy, ARA\&A, 43,531 (2005)

[2] P. Bonifacio, \& E. Caffau, Automatic abundance analysis of high resolution spectra, A\&A, 399,1183 (2003)

[3] E. Caffau, , L. Sbordone, H.-G. Ludwig, P. Bonifacio, M. Steffen, \& N. T. Behara, The solar photospheric abundance of hafnium and thorium. Results from $C O^{5} B O L D$ 3D hydrodynamic model atmospheres, A\&A, 483,591 (2008)

[4] P. François, E. Depagne, V. Hill et al., First Stars. III. A detailed elemental abundance study of four extremely metal-poor giant stars, A\&A, 403, 1105 (2003)

[5] B. Freytag, M. Steffen, B. Dorch, Spots on the surface of Betelgeuse - Results from new 3D stellar convection models, AN, 323, 213 (2002)

[6] R. L. Kurucz, CD-ROM 13, SAO, http://cfaku5.cfa.havard.edu/ (1993)

[7] R. L. Kurucz, ATLAS12, SYNTHE, ATLAS9, WIDTH9, et cetera, MSAIS, 8,14 (2005)

[8] A. McWilliam, Barium Abundances in Extremely Metal-poor Stars , AJ, 115, 1640 (1998)

[9] L. Sbordone, Kurucz's codes under GNU-Linux, MSAIS, 8,61 (2005)

[10] L. Sbordone, P. Bonifacio, F. Castelli, \& R. L. Kurucz, ATLAS and SYNTHE under Linux, MSAIS, $\mathbf{5}, 93(2004)$

[11] T. Sivarani, T. C. Beers, P. Bonifacio et al., First stars X. The nature of three unevolved carbon-enhanced metal-poor stars, A\&A, 459, 125 (2006)

[12] S. Wedemeyer, B. Freytag, M. Steffen, H. -G. Ludwig, H. Holweger, Numerical simulation of the three-dimensional structure and dynamics of the non-magnetic solar chromosphere, A\&A 414, 1121 (2004)

[13] D. G. York, J. Adelman, J. E. Anderson Jr., et al., The Sloan Digital Sky Survey: Technical Summary, AJ, 120,1579 (2000) 